\title{
A NEW CONCEPT IN DISTANCE GEOMETRY WITH APPLICATIONS TO SPHERICAL SUBSETS ${ }^{1}$
}

\author{
LEONARD M. BLUMENTHAL
}

1. Introduction. A semimetric space is a set of abstract elements (points) with a structure imposed by attaching to each pair $p, q$ of elements a non-negative real number $p q$ (distance) such that $p q=q p$ and $p q=0$ if and only if $p=q$. Some of the more important of these spaces (for example, euclidean, hyperbolic, and spherical spaces of $n$-dimensions) have the exceedingly useful property that the existence of a function mapping an arbitrary semimetric space congruently (that is, with preservation of distances) upon the space follows from the congruent embedding in the space of each set of $k$ points (not necessarily pairwise distinct) of the semimetric space. ${ }^{2}$ Such spaces are said to have congruence order $k$ with respect to all semimetric spaces-a term introduced by Menger, who proved that the $n$-dimensional euclidean space $E_{n}$ has this property with $n+3$ as the smallest value of $k .^{3}$

The importance of the possession by a given space of a congruence order is clear, since it evidently reduces the characterization problem of determining necessary and sufficient conditions for the congruent embedding of any semimetric space in the given one to the finite problem of finding under what conditions a semimetric set of $k$ points is congruently contained in the space. The desirability of having $k$ as small as possible is obvious.

After showing that $n+3$ is the minimum congruence order of the $n$-dimensional euclidean space $E_{n}$, Menger proved the more difficult theorem that if a semimetric space $S$ is not congruently contained in the $E_{n}$, but each set of $n+2$ of its points is congruent with $n+2$ points of the $E_{n}$, then $S$ consists of exactly $n+3$ points. Hence the congruent embedding in the $E_{n}$ of each set of $n+2$ points of a semimetric space $S$ insures that $S$ is congruently contained in $E_{n}$ whenever $S$ has more than $n+3$ points. This property was labelled "quasi congruence order

\footnotetext{
${ }^{1}$ Presented to the Society, April 12, 1940.

${ }^{2}$ See Chapter III of the author's Distance Geometries, University of Missouri Studies, vol. 13, 1938.

${ }^{3}$ The proof is given in Menger, Untersuchungen über allgemeine Metrik, Mathematische Annalen, vol. 100 (1928), pp. 75-163. The term "congruence order $n+3$ " describing this property was introduced in Bemerkungen zur zweiten Untersuchung über allgemeine Metrik, Proceedings of the Royal Academy, Amsterdam, vol. 30 (1927), pp. 1-5.
} 
$n+2 . " 4$ It is enjoyed by the $n$-dimensional hyperbolic space also, but not by the $n$-dimensional elliptic nor the $n$-dimensional spherical spaces. The notions of congruence and quasi congruence orders have been the principal ones employed in the metric characterizations of spaces.

Recent investigations of characterization problems for spherical subsets (that is, subsets of the surface of the sphere with geodesic (shorter arc) metric) have revealed the inadequacy of these two notions to describe the results obtained..$^{5}$ It is the purpose of this paper to present a general concept which systematizes all of these results, contains the older notions as two links of a chain of notions which occur in a special case, and which orders between these two links infinitely many other links. This new concept gives rise to a whole field of new problems in distance geometry and, besides, induces a re-examination of older, solved problems with a view towards a "better" solution. The meaning of the latter part of the above remark will become clear later.

2. $\sigma$-relative congruence indices. Let $S$ and $\sigma$ be two given semimetric spaces. Then $S$ has $\sigma$-relative congruence indices $(n, k)$ with respect to a given class $(\Sigma)$ of semimetric spaces provided any space $\Sigma$ of $(\Sigma)$ with more than $n+k$ pairwise distinct points is congruently contained in $S$ whenever each $n$ of its points (not necessarily pairwise distinct) is congruently contained in $\sigma$. It seems appropriate to call $\sigma$ a catalytic space of index $(n, k)$ of $S$ with respect to the class $(\Sigma)$, since it facilitates the desired reaction of congruent embedding of $\Sigma$ in $S$ but plays in general no part in the result itself, for $\Sigma$ is not necessarily embeddable in $\sigma$. If $\sigma=S$ the indices $(n, k)$ are called congruence indices of $S$ with respect to $(\Sigma)$. It is this case with which the present paper is principally concerned.

REMARK 1. A semimetric space $S$ has congruence indices $(n, 0)$ with respect to $(\Sigma)$ if and only if $S$ has congruence order $n$ with respect to $(\Sigma)$.

For suppose $S$ has congruence indices $(n, 0)$ with respect to $(\Sigma)$ and let $\Sigma$ be any space of $(\Sigma)$ with each $n$ of its points congruently contained in $S$. If $\Sigma$ contains more than $n$ pairwise distinct points, then $\Sigma$ is congruently contained in $S$ by hypothesis and definition. On the other hand, if $\Sigma$ consists of $n$ or fewer pairwise distinct points, the

\footnotetext{
4 The proof appears in Untersuchungen ïber allgemeine Metrik, loc. cit., but the term "quasi congruence order" is first defined in Menger, New foundations of euclidean geometry, American Journal of Mathematics, vol. 53 (1931), pp. 721-745.

${ }^{5}$ C. V. Robinson, Contributions to Distance Geometry, University of Missouri thesis.
} 
congruence of $\Sigma$ with a subset of $S$ is a trivial consequence of the congruent embedding in $S$ of each $n$ points of $\Sigma$. Hence $S$ has congruence order $n$ with respect to $(\Sigma)$.

Finally, if $S$ has congruence order $n$ with respect to $(\Sigma)$, then congruence of any space $\Sigma$ of $(\Sigma)$ follows from the congruent embedding in $S$ of each $n$ points of $\Sigma$, and the situation described by $S$ having congruence indices $(n, 0)$ with respect to $(\Sigma)$ evidently exists.

REMARK 2. A semimetric space $S$ has congruence indices $(n, 1)$ with respect to $(\Sigma)$ if and only if $S$ has the quasi congruence order $n$ with respect to $(\Sigma)$.

The proof is similar to that of Remark 1.

Thus the notions of congruence and quasi congruence orders correspond to particular $\sigma$-relative congruence indices in the special case $\sigma=S$.

Let $(n, k) \rightarrow\left(n^{\prime}, k^{\prime}\right)$ symbolize the statement, "if $S$ has congruence indices $(n, k)$ with respect to $(\Sigma)$, then $S$ has congruence indices $\left(n^{\prime}, k^{\prime}\right)$ with respect to $(\Sigma)$."

REMARK 3. If $n \leqq n^{\prime}$ and $n+k \leqq n^{\prime}+k^{\prime}$, then

$$
(n, k) \rightarrow\left(n^{\prime}, k^{\prime}\right) \text {. }
$$

The proof follows immediately from the definition of congruence indices.

Some special cases of Remark 3 are of interest. Thus, $(n, 1)$ $\rightarrow(n+1,0)$; that is, quasi congruence order $n$ implies congruence order $n+1$. Also $(n, 1) \rightarrow(n, 2) \rightarrow(n+1,1)$. This relation, which interpolates a property between quasi congruence order $n$ and quasi congruence order $n+1$ illustrates one of the advantages of the concept of congruence indices. Previous to its introduction, if a space $S$ were found not to have quasi congruence order $n$, the next step was to examine it for a higher quasi congruence order. Consideration was thus shifted from the congruent embedding in $S$ of sets of $n$ points to the more difficult problems concerning embedding in $S$ of sets of $n+1$ or more points. The possession by $S$ of congruence indices $(n, 2)$ implies quasi congruence order $n+1$, but, in addition, characterizes $S$ in terms of the congruent embedding in $S$ of each $n$ points of a semimetric space rather than each $n+1$ points.

In the light of the foregoing observations, any one of the congruence symbols $(n, k),(k=0,1,2, \cdots)$, is to be preferred to the symbol $(n+1,0)$, though only for $k=0,1$ is the implication $(n, k) \rightarrow(n+1,0)$ valid. Similarly, the symbols $(n, 1),(n, 2),(n, 3), \cdots$ are each preferable to $(n+1,1)$, though only the first two imply the latter. Thus, 
there is interpolated between quasi congruence orders $n$ and $n+1$ an infinity of symbols, each of which is to be preferred to quasi congruence order $n+1$. It is clear that the indices $(n, k)$ characterize a space only with respect to all semimetric spaces containing more than $n+k$ points, but this slight restriction of the class of comparison spaces is more than offset by the simplicity gained from keeping the number of points to be embedded as small as possible. In addition to the possibility of bettering old results (e.g., the possession of indices $(n, 2)$ where previously only $(n+1,1)$ and not $(n, 1)$ had been proved), new problems arise (e.g., the possibility of indices $(n, 3)$ when neither $(n, 1)$ nor $(n+1,1)$ exists for the space).

The above considerations suggest the following preferential (lexicographical) ordering of congruence indices. If $(n, k)$ and $\left(n^{\prime}, k^{\prime}\right)$ are two congruence symbols of $S$, that one is preferred for which (1) the first index is the smaller or (2) if $n=n^{\prime}$, then the preferred symbol has the smaller second index. That symbol $(n, k)$ of $S$ is best provided no symbol of $S$ is preferred to it. It is emphasized that this ordering is not that of logical implication. With respect to the latter criterion congruence symbols form only a partially ordered set (for example, the symbols $(n, 3)$ and $(n+1,1)$ are not logically comparable).

Since $(n, k) \rightarrow(n+k, 0), S$ has no congruence indices with respect to $(\Sigma)$ if $S$ has no congruence order with respect to $(\Sigma)$. Conversely, if $S$ has congruence order $m$ with respect to $(\Sigma)$, then by Remark 1 , $S$ has congruence indices with respect to $(\Sigma)$. Thus, $S$ possesses congruence indices if and only if $S$ has a congruence order.

3. Lemmas concerning pseudo-spherical sets. The $n$-dimensional spherical space $S_{n, r}$ of radius $r$ is the "surface" of a sphere of radius $r$ in a euclidean space of $n+1$ dimensions, with geodesic (shorter arc) metric. The $S_{n, r}$ has indices $(n+3,0)$ but not $(n+2,1)$, and there exist semimetric spaces of arbitrary power exceeding $n+2$ which are not contained congruently in $S_{n, r}$ though every set of $n+2$ points is congruently embeddable in $S_{n, r}$. Such spaces are called pseudo- $S_{n, r}$ sets. From the characterization of these spaces (given elsewhere) we take the following results : ${ }^{6}$

Property 1. Let $P$ be a pseudo- $S_{n, r}$ set of more than $n+3$ pairwise distinct points, no two of which have distance $\pi r$. If $p, q$ are any two distinct points of $P$, then

$$
\cos (p q / r)= \pm 1 /(n+1)
$$

${ }^{6}$ L. M. Blumenthal and G. R. Thurman, The characterization of pseudo-spherical sets, American Journal of Mathematics, vol. 62 (1940), pp. 835-854. 
Property 2. Let $P$ be a pseudo- $S_{n, r}$ set of $m$ pairwise distinct points

$$
p_{1}, p_{2}, \cdots, p_{m},
$$$$
m>n+3
$$

no two of which have distance $\pi r$, and consider the determinant

$$
\Delta_{m}\left(p_{1}, p_{2}, \cdots, p_{m}\right)=\left|\cos \left(p_{i} p_{j} / r\right)\right|, \quad i, j=1,2, \cdots, m,
$$

of the set. A part from the elements of the first and jth rows, $(j=2,3, \cdots, m)$, either each element in the first column of $\Delta_{m}$ is equal to the corresponding element in the jth column, or each element in the first column is the negative of the corresponding element in the jth column. If the first column of $\Delta_{m}$ contains at least one positive element different from the element 1 , then apart from the elements in the first and second rows, each element in the second column is the negative of the corresponding element in the first column. Due to the symmetry of $\Delta_{m}$ and the first part of this property, the sign of every element of $\Delta_{m}$ is then determined. ${ }^{7}$

Property 3. If $P$ is a pseudo- $S_{n, r}$ set of more than $n+3$ pairwise distinct points, no two of which have distance $\pi r$, then every set of $m$ points of $P,(m \geqq n+3)$, is a pseudo- $S_{n, r}$ set.

Lemma 1. Apart from the labelling, there is a unique pseudo- $S_{n, r}$ set of $2 n+2$ pairwise distinct points, $(n>1)$, free from diametral pointpairs (that is, pairs of points with distance $\pi r$ ), which does not contain an equilateral set of $n+2$ points (that is, a set of $n+2$ points with all distances equal). ${ }^{8}$

Proof. Let $P$ be any pseudo- $S_{n, r}$ set of $2 n+2$ points, no two of which have distance $\pi r$. Since $n>1,2 n+2>n+3$, and the determinant $\Delta_{2 n+2}$ of $P$ has Properties 1, 2 listed above. To prove the lemma it suffices to show that apart from a (symmetric) shifting of rows and columns, there is a single distribution of the signs in $\Delta_{2 n+2}$ which will not yield a principal minor of order $n+2$ with all elements outside the principal diagonal negative. (Since each set of $n+2$ points of $P$ is congruently contained in $S_{n, r}$, an $(n+2)$-nd order principal minor of $\Delta_{2 n+2}$ with all positive elements is impossible. Thus an equilateral $(n+2)$-tuple has each distance equal to $r \cdot \cos ^{-1}-1 /(n+1)$.)

Applying Property 2, a brief consideration of $\Delta_{2 n+2}$ shows that the first column of the determinant must contain exactly $n+1$ positive elements (apart from 1) and an appropriate (symmetric) shifting of

${ }^{7}$ See L. M. Blumenthal, Metric methods in determinant theory, American Journal of Mathematics, vol. 61 (1939), pp. 912-922.

${ }^{8}$ This lemma is not valid for $n=1$. 
rows and columns gives (schematically):

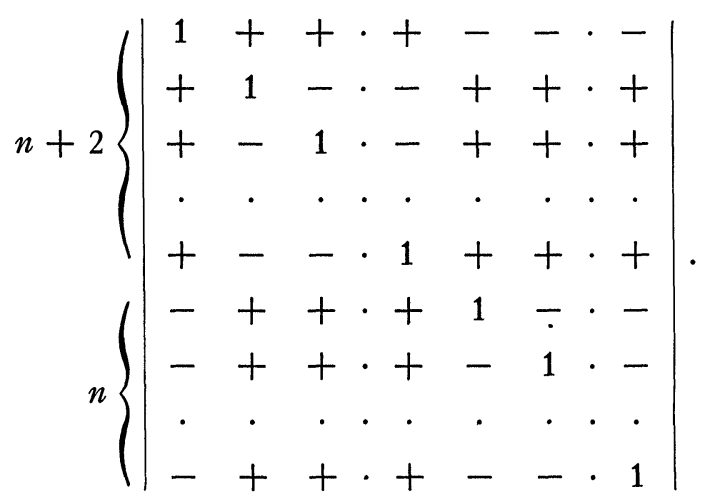

Corollary. Every pseudo- $S_{n, r}$ set of $2 n+2$ pairwise distinct points, $(n>1)$, no two with distance $\pi r$, contains an equilateral $(n+1)$-tuple with each distance equal to $r \cdot \cos ^{-1}-1 /(n+1)$.

For the only such set without an equilateral $(n+2)$-tuple is, apart from labelling, the set exhibited in the determinant of Lemma 1. Deleting the 2 nd, $3 r d, \cdots,(n+2)$-nd rows and columns of this determinant, one obtains the determinant of a set of the kind specified in the corollary. Another such set is given by deleting the 1st, $(n+3)$-rd, $\cdots,(2 n+2)$-nd rows and columns.

Lemma 2. Each pseudo- $S_{n, r}$ set $P$ of more than $2 n+2$ pairwise distinct points, $(n>1)$, no two of which have distance $\pi r$, contains an equilateral $(n+2)-$ tuple. $^{9}$

Proof. Since $n>1,2 n+2>n+3$, and hence $P$ contains more than $n+3$ pairwise distinct points. By Property 3 , each set $p_{1}, p_{2}, \cdots, p_{2 n+3}$ of $2 n+3$ points of $P$ is a pseudo- $S_{n, r}$ set. It suffices to show that these points contain an equilateral $(n+2)$-tuple. Again by Property 3 , the points $p_{1}, p_{2}, \cdots, p_{2 n+2}$ form a pseudo- $S_{n, r}$ set of more than $n+3$ points, and according to Lemma 1 either these points contain an equilateral $(n+2)$-tuple or they constitute a set of the kind exhibited in the proof of that lemma.

In the latter case, consider the determinant $\Delta_{2 n+3}$ of the points $p_{1}, p_{2}, \cdots, p_{2 n+3}$. The principal minor of order $2 n+2$ in the upper left-hand corner of $\Delta_{2 n+3}$ is the determinant of Lemma 1. If the element in the last row and first column of $\Delta_{2 n+3}$ is positive, then

${ }^{9}$ We suppose $n>1$ since Lemma 1 is applied. It is easily shown that Lemma 2 is valid for $n=1$. 
the principal minor of order $n+2$ obtained by deleting the 1 st, $(n+4)$-th, $\cdots,(2 n+3)$-rd rows and columns of $\Delta_{2 n+3}$ has all its elements outside the principal diagonal negative, and hence the corresponding $(n+2)$-tuple is equilateral. If, on the other hand, the element in the last row and first column of $\Delta_{2 n+3}$ is negative, deleting the $2 \mathrm{nd}, 3 \mathrm{rd}, \cdots,(n+2)$-nd rows and columns yields the determinant of an equilateral $(n+2)$-tuple.

4. Applications to spherical subsets. The subsets of the $S_{n, r}$ to which the foregoing is applied in this paper are spherical caps $\kappa_{n, \rho}$ of spherical radius $\rho, 0<\rho \leqq \pi r / 2$ (that is, the locus of points of $S_{n, r}$ whose distance from a fixed point of $S_{n, r}$ is less than or equal to $\rho$ ).

Theorem 1. Let $\sigma$ denote the spherical cap (n-dimensional hemisphere) $\kappa_{n, \pi r / 2}, n>1$, with base circle removed. Then $S_{n, r}$ has $\sigma$-relative congruence indices $(n+2, n)$ with respect to all semimetric spaces $(\Sigma) .{ }^{10}$

Proof. We must show that each semimetric space $\Sigma$ containing more than $2 n+2$ pairwise distinct points and having each $n+2$ of them congruent with $n+2$ points of $\sigma$ is congruently contained in $S_{n, r}$. Suppose this is not the case. Then $\Sigma$ is a pseudo- $S_{n, r}$ set of more than $2 n+2$ points, no two of which have distance $\pi r$ (since each $n+2$ points of $\Sigma$ are congruently contained in $\sigma$ ) and hence, by Lemma 2, $\Sigma$ contains an equilateral set of $n+2$ points with each of the $\frac{1}{2}(n+1)(n+2)$ mutual distances equal to $r \cdot \cos ^{-1}-1 /(n+1)$. But this is not possible, for such an $(n+2)$-tuple is clearly not contained in $\sigma .^{11}$ This contradiction yields the theorem. Obvious examples show that $\Sigma$ is not necessarily contained in $\sigma$.

Thus the open $n$-dimensional hemisphere is a catalytic set of index $(n+2, n)$ of the containing sphere.

THEOREM 2. Let $\kappa_{n, \rho}, n>1$, be an $n$-dimensional spherical cap of $r a$ dius $\rho,(\rho<\pi r / 2)$. Then $\kappa_{n, \rho}$ has congruence indices $(n+2, n)$ with respect to all semimetric spaces $(\Sigma)$.

Proof. Let $\Sigma$ be any semimetric space containing more than $2 n+2$ points, each $n+2$ of which are congruently contained in $\kappa_{n, \rho}$. Then it

\footnotetext{
${ }^{10}$ Since any $\operatorname{arc} \kappa_{1, \rho}, \rho \leqq \pi r / 2$, of a circle $S_{1, r}$ is evidently congruent with a straight line segment (which has indices $(3,1)$ with respect to $(\Sigma)$ ), Theorems 1,2 of this section hold for $n=1$.

${ }^{11}$ In the contrary case there exist $n+2$ positive numbers $b_{1}, b_{2}, \cdots, b_{n+2}$ such that the $(n+3)$ rd order determinant obtained by bordering the determinant of the equilateral set by these numbers (with 1 in the intersection of the bordering row and column) is zero. This yields quite easily $\sum_{i=1}^{n+2} b_{i}=0$, which is impossible.
} 
follows from Theorem 1 that $\Sigma$ is congruently contained in $S_{n, r}$. But it has been shown that with respect to subsets of $S_{n, r}$, the cap $\kappa_{n, \rho}$, $(\rho<\pi r / 2)$, has congruence indices $(n+2,0) .{ }^{12}$ Hence $\Sigma$ is congruently contained in $\kappa_{n, \rho}$, and the theorem is proved.

REMARK 1. Since $(n+2, n) \rightarrow(2 n+1,1)$, the cap $\kappa_{n, \rho},(\rho<\pi r / 2)$, has quasi congruence order $2 n+1$ with respect to semimetric spaces. As $n$ increases, however, the indices on the left-hand side of the above implication tend to place conditions on only half the number of points that must be isometrically embedded if the characterization is given in terms of the indices on the right-hand side of the implication.

Theorem 3. The cap $\kappa_{n, \rho},\left(\rho<r \cdot \cos ^{-1} 1 /(n+1)\right), n>1$, has congruence indices $(n+2, n-1)$ with respect to all semimetric spaces $(\Sigma) .{ }^{13}$

Proof. If $\Sigma$ is any semimetric space of more than $2 n+1$ pairwise distinct points, each $n+2$ of which are embeddable in $\kappa_{n, \rho}$, $\left(\rho<r \cdot \cos ^{-1} 1 /(n+1)\right)$, then $\Sigma$ is congruently contained in $S_{n, r}$, for an assumption to the contrary implies that $\Sigma$ is a pseudo- $S_{n, r}$ set of at least $2 n+2$ pairwise distinct points (no two with a distance $\pi r$ ). Then by the corollary to Lemma $1, \Sigma$ contains an equilateral $(n+1)$-tuple with each distance equal to $r \cdot \cos ^{-1}-1 /(n+1)$. Such an $(n+1)$-tuple is not embeddable in any cap of radius $\rho<r \cdot \cos ^{-1} 1 /(n+1)$. Since, now, $\Sigma$ is congruently contained in $S_{n, r}$ and each cap $\kappa_{n, \rho}$ of radius $\rho<\pi r / 2$ has congruence indices $(n+2,0)$ with respect to subsets of $S_{n, r}$, it follows that $\Sigma$ is congruently contained in $\kappa_{n, \rho},\left(\rho<r \cdot \cos ^{-1} 1 /(n+1)\right)$.

REMARK 1. For $n=2$, the cap $\kappa_{2, \rho},\left(\rho<r \cdot \cos ^{-1} 1 / 3\right)$, has indices $(4,1)$; that is, quasi congruence order 4 with respect to all semimetric spaces. Thus spherical caps with radii less than $r \cdot \cos ^{-1} 1 / 3$ behave like the euclidean plane in this important respect. The indices $(4,1)$ cannot be improved no matter how small $\rho$ is taken.

REMARK 2. For $n=3$, the indices $(5,2)$ of the cap $\kappa_{3, \rho}, \rho<r \cdot \cos ^{-1} 1 / 4$, given by Theorem 3 , can be bettered. A brief consideration shows that every pseudo- $S_{3, r}$ set of more than six points (no two with distance $\pi r$ ) contains at least one equilateral set of four points with mutual distances equal to $r \cdot \cos ^{-1}-1 / 4$. A proof quite similar to that of Theorem 3 demonstrates that $\kappa_{3, \rho}, \rho<r \cdot \cos ^{-1} 1 / 4$, has the indices $(5,1)$ and hence behaves like the euclidean three-dimensional spaces in this respect.

${ }^{12}$ C. V. Robinson, loc. cit.

${ }^{13}$ This theorem is not valid for $n=1$. 
REMARK 3. If $n=4$, the indices $(6,3)$ given by Theorem 3 for the cap $\kappa_{4, \rho},\left(\rho<r \cdot \cos ^{-1} 1 / 5\right)$, cannot be reduced to $(6,1)$ by a simple argument analogous to that presented in the preceding remark. ${ }^{14}$ This arises from the fact that there exists a pseudo- $S_{4, r}$ set of eight points (no two diametral) that does not contain an equilateral quintuple with mutual distances equal to $r \cdot \cos ^{-1}-1 / 5$. Such a pseudo- $S_{4, r}$ set is represented schematically by

$$
\left|\begin{array}{cccccccc}
1 & + & + & + & + & - & - & - \\
+ & 1 & - & - & - & + & + & + \\
+ & - & 1 & - & - & + & + & + \\
+ & - & - & 1 & - & + & + & + \\
+ & - & - & - & 1 & + & + & + \\
- & + & + & + & + & 1 & - & - \\
- & + & + & + & + & - & 1 & - \\
- & + & + & + & + & - & - & 1
\end{array}\right| .
$$

There is no fifth-order principal minor of this determinant with all elements outside the principal diagonal negative.

The problems of (1) finding the best indices for caps $\kappa_{n, \rho}$, $\rho<r \cdot \cos ^{-1} 1 /(n+1),(n>1)$, and (2) ascertaining for each $n$ the $n$-dimensional cap of maximum spherical radius $\rho$ that, like the $n$-dimensional euclidean space, has congruence indices $(n+2,1)$, will be investigated in a later paper.

UNIVERSITY OF MISSOURI

\footnotetext{
${ }^{14}$ It is easily shown that the indices $(6,3)$ can be reduced to $(6,2)$. Whether the cap $\kappa_{4, \rho},\left(\rho<r \cos ^{-1} \frac{1}{5}\right)$, does behave like the four-dimensional euclidean space with regard to the symbol $(6,1)$ is not known.
} 